\title{
Advanced Imaging in Biology and Medicine: Technology, Software Environment, Applications
}

C.W. Sensen and B. Hallgrimsson, eds.

Berlin, Germany: Springer-Verlag, 2009, 445 pages, $\$ 219$

The purpose of this book is to provide a broad sampling of the rapidly expanding field of advanced imaging in medicine and biology. The book is aimed toward radiologists, medical physicists, scientists in the pharmacologic and biologic industries, and computer software and hardware specialists in this field. Some of the material presented would benefit dermatologists, ophthalmologists, pathologists, cardiologists, veterinarians, geneticists, and virtual reality developers. Trainees in all these specialties would also benefit from this book.

The book is divided into 3 parts and 20 chapters.

Part I consists of 10 chapters that describe the various imaging modalities: micro-CT, experimental MRI, 3-dimensional ultrasound, laser scanning, optical coherence tomography, mass spectroscopy-based tissue imaging, optical projection tomography, ultrastructure imaging, and an introduction to medical imaging modalities. One chapter is dedicated to imaging and evaluation of live tissues at the microscopic level.

Part II is dedicated to software and includes 5 chapters: volume visualization using virtual reality, surface render- ing, CAVEman (an object-oriented model of the human body), image-based finite element analysis, and geometric morphometrics.

Five chapters constitute Part III. These chapters describe applications in audiology, molecular MRI, genomic data visualization, anatomic imaging, and postgenomic biology and functional measures of therapy.

The chapters are well written, easy to follow, and provide comprehensive references. Some of these chapters may overwhelm certain readers but stimulate those readers to further pursue these topics. Two chapters that stand out are those describing optical projection imaging and imaging evaluating live tissues at the microscopic level.

The authors are commended for presenting a complex field in a nice, compact, but stimulating book.

Aurelio Matamoros, Jr.

M.D. Anderson Cancer Center

Houston, Texas 